\title{
QUANTUM STOCHASTIC CALCULUS AND THE DYNAMICAL STARK EFFECT
}

\author{
PAUL ROBINSON* \\ Institute of Mathematics, University of Hull, Hull, United Kingdom \\ and \\ HANS MAASSEN** \\ Institute of Mathematics, University of Nijmegen, 6525 ED Nijmegen, the Netherlands
}

(Received November 6, 1990)

\begin{abstract}
Using (non-adapted) quantum stochastic calculus as our main tool we calculate the dynamical Stark effect, i.c., the splitting of the fluorescent spectrum of a two-level atom in a strong laser beam. We introduce the scattering operator for this system, and establish a well-known relation between the scattered spectrum and the autocorrelation function of the atom. We obtain an expression for the spectrum which is in agreement with that obtained by Mollow in. 1969, and generalises it to arbitrary temperaturcs.
\end{abstract}

\section{§o. Introduction}

In this paper we want to illustrate that quantum stochastic calculus forms a suitable framework to calculate and discuss interesting phenomena in quantum optics. As an example we consider the case of the dynamical Stark effect. Some other cases-notably the electron shelving effect-have been treated by Barchielli [1, 2].

The phenomenon we shall consider here occurs when an atom is put into an intense laser beam, tuned precisely to one of its transition frequencies: the atom becomes fluorescent; its emitted spectrum consists of three broadened peaks with height ratio 1:3:1 and width ratio $3: 2: 3$. The distance of the side peaks from the central one increases with the amplitude of the laser beam. Now, the dependence of the position of spectral lines on the amplitude of a-constant-external field is known as the "Stark effect". Hence, the phenomenon described above was given the name of dynamical or resonam Stark cffect. It was predicted in 1969 by Mollow [15] on the basis of a quantum Masier equation. $A$ debate ensued as to the correctness of his conclusions and lasted until the prediction was experimentally verified by Schuda, Stroud and Hercher in 1974 [17].

The participants in the theoretical discussion held in the arly and mid seventies an be roughy divided into two camps: those employing the perturbative sachnigues of cuantum

\footnotetext{
* P. Robinson is pleased to ackuowledge the findncial surport of the Netherlands Organisation of Sacntific Resaarch NWO. provided under the Royal Sociciys European Exchange programme.

$*$ H. Maassen acknowledges support from the Netherlands Organisation of Seientific Rescarch VWo
} 
electrodynamics $[5,18]$ and those using the probabilistic language of quantum Master equations $[4,15]$.

The present treatment unifies these two approaches in the mathematical framework of quantum stochastic calculus. An extra feature is the inclusion of a temperature $\beta^{-1}$ in the model, dictated by mathematical convenience [12].

Our starting point is a semigroup of transition operators $T_{t},(t \geq 0)$ on the algebra $M_{2}$ of all $2 \times 2$-matrices. This semigroup describes the approach to the thermal equilibrium state $\varphi_{\beta}$ at temperature $\beta^{-1}$ of an atom possessing two energy levels.

By coupling the atom to a quantum noise source we construct a stationary quantum Markov process having precisely these transition operators. In one imposes the requirements that the external noise be a Bose field, and the quantum Markov process be minimal, then the latter is uniquely determined. Is is called the minimal Rose dilation of $\left(M_{2}, T_{t}, \varphi_{B}\right)[8,12]$.

Since this dilation is uniquely determined, any other reversible dynamical model which couples $\left(M_{2}, T_{t}, \varphi_{3}\right)$ to some Bose ficld nccessarily contains this minimal Bose dilation as a subsystem. Hence without deriving our model from an explicit Schrödinger equation (by performing some Markovian limit) we may safely assume it to be physically correct.

Then, as we are interested in the change which field observables undergo on passing the atom, we study the scattering operator associated to the Markov process [9] we constructed. The fluorescent spectrum as determined by the scattering operator, can be conveniently expressed in terms of the autocorrelation function of the atom's excitation operator in a scattered state. Quantum stochastic calculus gives ready access to this autocorrelation function.

We employ a version of quantum stochastic calculus based on integral kernels [12, 13]. This has the advantage that non-adapted processes can be dealt with as easily as adapted ones. In particular, an Itô formula for non-ddapted processes is presented. Moreover the kernel formulation of quantum stochastic calculus forges a link between the quantum electrodynamical and probabilistic treatments of this problem. In this context it is illuminating to compare our formula (1.2) with the series expansion studied by Mollow [16].

The paper is organised as follows. In Section 1 we introduce kernel processes with values in a $C^{*}$-algebra, and their associated Itô formula. We solve explicitly a relevant quantum stochastic differential equation. In Section 2 we construct the Bose dilation of $\left(M_{2}, T_{t}, \varphi_{3}\right)$. In Section 3 the scattering operator is introduced, in particular its action on modes of the Bose field. A link is established in Section 4 between the scattered spectrum of a given square-integrable input field and the autocorrelation function of the atom's excitation operator. A well-known differential equation for this correlation function is proved. In Section 5 we extend the validity of the differential equation beyond the square-integrable input fields by an approximation theorem. We then calculate explicitly the fluorescence spectrum in Section 6 for the case of a plane wave input field, reproducing (and slightly extending) Mollow's result.

\section{$\S 1$. The kernel calculus of Bose noise}

Kernels, kernel processes and their associated differential and integral calculus are 
discussed in detail in [12]; a brief, but essentially self-contained account can be found in [10] and [14].

Let $\nabla_{0}$ denote the metric space of finite configurations $\sigma$ of + 's and -'s on the line. We shall treat elements $\sigma$ of $\nabla_{0}$ as finite "charged" subsets of $\mathbf{R}$. Physically we will view the +'s and -'s as photons added to, and removed from, a thermal background respectively. For analytic reasons it will be convenient to consider the completion $\nabla$ of $\nabla_{0}$. In $\nabla$ configurations are allowed where photons coincide. In such "boundary" configurations account has to be taken of the order of the charges in a single point. Different orders stand for different limits of configurations in $\nabla_{0}$.

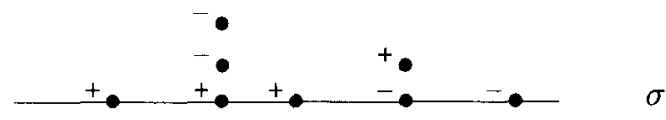

Write $\sigma^{+}, \sigma^{-}$for the positive and negative parts of $\sigma$ and $|\sigma|$ for the number of elements in $\sigma$; then we can write,

$$
\nabla=\bigcup_{j, k=0}^{\infty} \nabla^{j, k}, \quad \text { where } \quad \nabla^{j, k}=\left\{\sigma \in \nabla|| \sigma^{+}|=j,| \sigma^{-} \mid=k\right\} .
$$

Now let $a>b>0$ and define a measure $\mu=\mu_{a, b}$ on $\nabla$ by,

$$
\mu(\{\phi\})=1 ;\left.\quad \mu\right|_{\nabla^{j, k}}=a^{j} b^{k} \lambda_{j+k},
$$

where $\phi$ denoted the empty set and $\lambda_{n}$ is $n$-dimensional Lebesgue measure.

Let $\mathcal{A}$ be a $C^{*}$-algebra and denote by $\mathcal{K}(\mathcal{A})$ the class of smooth $\mathcal{A}$-valued functions on $\nabla$ [12]. The operation $*: \mathcal{K}(\mathcal{A}) \times \mathcal{K}(\mathcal{A}) \rightarrow \mathcal{K}(\mathcal{A})$ and the involution ${ }^{*}: \mathcal{K}(\mathcal{A}) \rightarrow \mathcal{K}(\mathcal{A})$ defined by,

$$
\begin{gathered}
x * y(\sigma)=\sum_{\alpha \subset \sigma} \int_{\nabla} x(\widetilde{\omega} \cup \alpha) y(\omega \cup \bar{\alpha}) \mu(d \omega), \\
x^{*}(\omega)=x(\widetilde{\omega})^{+},
\end{gathered}
$$

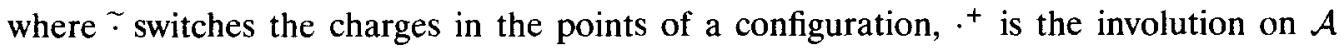
and the sum is over all partitions of $\sigma$ into disjoint subsets $\alpha$ and $\bar{\alpha}$, render $\mathcal{K}(\mathcal{A})$ an associative *-algebra. (Proposition 2.7 of [12]).

Given a smooth $\mathbf{C}$-valued kernel $x \in \mathcal{K}(\mathbf{C})$, the map $X: y \mapsto x * y$ defines an operator on $K(\mathbf{C})$. If $x$ is a Weyl kernel $w_{f}$, defined by

$$
w_{f}(\sigma)=\exp \left(-\frac{1}{2}(a+b)\|f\|^{2}\right) \prod_{s \in \sigma^{+}} f(s) \prod_{t \in \sigma^{-}}(-\overline{f(t)}), \quad\left(f \in C_{0}^{\infty}(\mathbf{R}, \mathbf{C})\right),
$$

then $X$ extends to a unitary operator $W(f)$ on $\mathcal{H}_{a, b}:=L^{2}\left(\nabla, \mu_{a, b}\right)$. The operators $W(f)$ satisfy Weyl's version of the Canonical Commutation Relations:

$$
W(f+g)=\mathrm{e}^{i(a-b) \operatorname{Im}\langle f, g\rangle} W(f) W(g) .
$$

The correspondence $f \mapsto W(f)$ extends weak-*-continuously to all $f \in l^{2}(\mathbf{R})$. 
Let $\delta_{\phi} \in \mathcal{H}_{a, b}$ be the vector given by

$$
\delta_{\phi}(\sigma)= \begin{cases}1 & \text { if } \sigma=\phi \\ 0 & \text { otherwise }\end{cases}
$$

In the associated vector state $\chi_{a, b}: X \rightarrow\left\langle\delta_{\phi}, X \delta_{\phi}\right\rangle=x(\phi)$ the Weyl operators have the expectations

$$
\chi_{u, b}(W(f))=u_{f}(\phi)=\mathrm{e}^{-\frac{1}{2}(a+b)|| f \|^{2}} .
$$

In view of the above it is natural to put $a-b=1$ and introduce a temperature $\beta^{-1}$ by $\mathrm{e}^{3}-a / b$, so that

$$
a=\frac{\mathrm{e}^{\beta}}{\mathrm{e}^{\beta}-1} \text { and } b=\frac{1}{\mathrm{e}^{\beta}-1} .
$$

We shall henceforth speak of $\mathcal{H}_{\beta}$ instead of $\mathcal{H}_{a, b}, \chi_{\beta}$ instead of $\chi_{a, b}$, etc.

The von Neumann algebra $\mathcal{N}_{\beta}$ generated by the $W(f)$ on $\mathcal{H}_{\beta}$, consists of all operators $y \mapsto x * y$ which are bounded. We call $\mathcal{N}_{\beta}$ the algebra of Bose noise at inverse temperature $\beta$, and $\chi_{3}$ the associated equilibrium state on $\mathcal{N}_{3}$.

A smooth kernel process is a map $x: \mathbf{R} \rightarrow \mathcal{K}(\mathcal{A})$ such that the map $(\sigma, t) \mapsto x_{t}(\sigma)$ is smooth. Let $\mathcal{P}(\mathcal{A})$ denote the collection of such processes; then under the pointwise operation

$$
(x * y)_{t}=x_{t} * y_{t}
$$

it forms an associative ${ }^{*}$-algebra as well.

We define the following operations on $\mathcal{P}(\mathcal{A})$,

$$
\begin{aligned}
& \left(J_{\varepsilon}^{\downarrow} x\right)_{t}(\sigma):=\lim _{s \downarrow t} x_{s}\left(\sigma \cup\left\{t^{\varepsilon}\right\}\right), \quad \varepsilon= \pm 1, \\
& \left(J_{\varepsilon}^{\uparrow} x\right)_{t}(\sigma):=\lim _{s \uparrow t} x_{s}\left(\sigma \cup\left\{t^{\varepsilon}\right\}\right), \quad \varepsilon= \pm 1, \\
& (D x)_{t}(\sigma):= \begin{cases}\frac{d x_{t}(\sigma)}{d t} & \text { where it exists, } \\
0 & \text { otherwise. }\end{cases}
\end{aligned}
$$

Let $\mathcal{P}^{l}(\mathcal{A})$ denote the * -algebra of smooth processes $x$ for which $D x \in \mathcal{P}(\mathcal{A})$. The following result will be our main computational tool.

Proposition 1.1 (Quantum Itô's formula). For all processes $x, y \in \mathcal{P}^{1}(\mathcal{A})$,

(a) $J_{\varepsilon}^{\downarrow}(x * y)=\left(J_{\tilde{\varepsilon}}^{\downarrow} x\right) * y+x *\left(J_{\varepsilon}^{\downarrow} y\right),(\varepsilon= \pm 1)$, and similarly for $J_{\varepsilon}^{\uparrow}$;

(b) $D(x * y)=D x * y+x * D y+a\left(J_{-}^{\downarrow} x * J_{+}^{\dagger} y-J_{-}^{\uparrow} x * J_{+}^{\dagger} y\right)+b\left(J_{+}^{\downarrow} x * J_{-}^{\downarrow} y-J_{+}^{\uparrow} x * J_{-}^{\uparrow} y\right)$.

This formula is an easy generalisation of the quantum Itô formula for adapted processes $[7,11,12]$. We call a process adapted if it is supported on the adapted simplex

$$
\nabla_{\mathrm{ad}}=\left\{(\sigma, t) \in \nabla \times \mathbf{R}^{+}|\sigma \subset| 0, t \mid\right\} .
$$

By $\mathcal{P}_{\text {aul }}(\mathcal{A})$ we denote the *algebra of adapted processes.

If $x \in \mathcal{P}_{\mathrm{ad}}(\mathcal{A})$ then $\left(J_{\varepsilon}^{\dagger} x\right)=0,(\varepsilon= \pm 1)$, since for $s<t$ the configuration $\sigma \cup\left\{t^{\varepsilon}\right\}$ is not contained in $[0, s] ;\left(J_{\varepsilon}^{\downarrow} x\right)$ is the stochastic derivative, and $D x$ the forward derivative of process $x$. 
THEOREM 1.2 [12]. Let $L_{+}, L_{-}$and $L_{0}$ be bounded linear maps on $\mathcal{A}$. Then the unique adapted solution $y \in \mathcal{P}_{\mathrm{ad}}^{1}(\mathcal{A})$ to the system

$$
J_{+}^{\downarrow} y=L_{+}(y) ; \quad J_{-}^{\downarrow} y=L_{-}(y) ; \quad D y=L_{0}(y) ; \quad y_{0}(\phi)=y_{0} \in \mathcal{A} ;
$$

is given explicitly by,

$$
\begin{gathered}
y_{t}(\sigma)=P\left(t-s_{n}\right) L_{\varepsilon_{n}} P\left(s_{n}-s_{n-1}\right) \ldots P\left(s_{2}-s_{1}\right) L_{\varepsilon_{1}} P\left(s_{1}\right)\left(y_{0}\right), \\
\sigma=\left\{s_{j}^{\varepsilon_{j}}\right\}_{j=1}^{n} ; \quad 0 \leq s_{1} \leq s_{2} \leq \ldots \leq s_{n} \leq t,
\end{gathered}
$$

where $P(t)=\mathrm{e}^{t L_{0}},(t \geq 0)$.

\section{§2. The Wigner-Weisskopf atom}

Let $M_{2}$, the algebra of $2 \times 2$-matrices, stand for the observables of an atom possessing only two energy levels: an upper level 1 and a lower level 2 . If the atom jumps downward or upward between its two states with transition rates $a$ and $b$ respectively, then the irreversible evolution of the atom is described by the semigroup $\left(T_{t}: M_{2} \rightarrow M_{2}\right)_{t \geq 0}$ of completely positive maps $T_{t}=\mathrm{e}^{t L_{0}}$ with

$$
L_{0}:\left(\begin{array}{ll}
x_{11} & x_{12} \\
x_{21} & x_{22}
\end{array}\right) \mapsto\left(\begin{array}{cc}
a\left(x_{22}-x_{11}\right) & \left(i \omega_{0}-\frac{1}{2}(a+b)\right) x_{12} \\
-\left(i \omega_{0}+\frac{1}{2}(a+b)\right) x_{21} & -b\left(x_{22}-x_{11}\right)
\end{array}\right) .
$$

Here $\omega_{0}$ is the natural transition frequency of the atom. Note that for $x \in M_{2}$,

$$
L_{0}(x)=i\left[h_{0}, x\right]-\frac{1}{2} a\left(v^{+} v x-2 v^{+} x v+x v^{+} v\right)-\frac{1}{2} b\left(v v^{+} x-2 v x v^{+}+x v v^{+}\right),
$$

where $v=\left(\begin{array}{ll}0 & 0 \\ 1 & 0\end{array}\right)$ and $v^{+}=\left(\begin{array}{ll}0 & 1 \\ 0 & 0\end{array}\right)$ are the annihilation and creation operators of atomic excitations respectively, and $h_{0}$ denotes the matrix $\left(\begin{array}{cc}\omega_{0} & 0 \\ 0 & 0\end{array}\right)$.

For the above model,

$$
\lim _{t \rightarrow \infty} T_{t}(x)=\varphi_{\beta}(x) \cdot \mathbf{1}, \quad\left(x \in M_{2}\right),
$$

where 1 is the unit matrix in $M_{2}$ and $\varphi_{\beta}$ is the thermal equilibrium state on $M_{2}$ given by,

$$
\varphi_{\beta}(x)=\frac{a x_{22}+b x_{11}}{a+b} .
$$

This quantum dynamical semigroup describes the approach to thermal equilibrium of a two-level atom coupled to a heat bath at temperature $\beta^{-1}$. The system $\left(M_{2}, T_{t}, \varphi_{\beta}\right)$ is known as the Wigner-Weisskopf atom [20].

We shall now embed $M_{2}$ with its state $\varphi_{\beta}$ into the von Neumann algebra $M_{2} \otimes \mathcal{N}_{\beta}$ with state $\widehat{\varphi}_{\beta}:=\varphi_{\beta} \otimes \chi_{\beta}$ and construct a group of $\widehat{\varphi}_{\beta}$-preserving automorphisms $\left\{\widehat{T}_{t}\right\}_{t \in \mathbf{R}}$ on $M_{2} \otimes \mathcal{N}_{\beta}$ such that the diagram

$$
\begin{array}{ccc}
M_{2} \otimes \mathcal{N}_{3} & \stackrel{\widehat{T}_{t}}{\longrightarrow} & M_{2} \otimes \mathcal{N}_{\beta} \\
i \uparrow & & \rfloor_{M_{2}} i d \otimes \chi_{\beta} \\
M_{2} & \stackrel{\widehat{T}_{t}}{\longrightarrow} & M_{2}
\end{array}
$$


commutes, where $i: x \mapsto x \circledast \mathbf{1}_{\mathcal{N}}$.

Let $u \in \mathcal{P}_{\mathrm{ad}}\left(M_{2}\right)$ be the adapted solution of the system,

$J_{+}^{\downarrow} u=v u ; \quad J_{-}^{\downarrow} u=-v^{+} u ; \quad D u=\left(i h_{0}-\frac{1}{2}\left(a v^{+} v+b v v^{+}\right)\right) u ; \quad u_{0}=\delta_{\phi} \cdot \mathbf{1}$.

By Proposition 8.5 of [12] the solution defines a family of unitary maps $U_{t}: y \mapsto u_{t} * y$ on $M_{2} \otimes \mathcal{H}_{\beta}$, where we view $M_{2}$ as a Hilbert space under the inner product $\langle x, y\rangle_{\beta}=\varphi_{\beta}\left(x^{+} y\right)$.

Let $\sigma_{t}$ denote the right shift through $t \in \mathbf{R}$ over $\mathcal{N}_{\beta}$ (or $\mathcal{H}_{\beta}$ ). Then the family of maps $\widehat{T}_{t}: M_{2} \otimes \mathcal{N}_{\beta} \rightarrow M_{2} \otimes \mathcal{N}_{\beta}$ given by,

$$
\begin{gathered}
\widehat{T}_{t}(X)=U_{t}^{*}\left(\mathrm{id} \otimes \sigma_{t}\right)(X) U_{t}, \quad(t \geq 0), \\
\widehat{T}_{t}=\left(\widehat{T}_{-t}\right)^{-1}, \quad(t<0),
\end{gathered}
$$

meets all requirements ([12], Lemmas 9.3 and 9.4). Because $\widehat{T}_{t}$ preserves $\widehat{\varphi}_{\beta}$, $\widehat{T}_{t}$ determines a unitary group on the Hilbert space $M_{2} \otimes \mathcal{H}_{3}$ by continuous extension of the operators

$$
x=X \delta_{\phi} \mapsto \widehat{T}_{t}(X) \delta_{\phi}, \quad(t \in \mathbf{R}),
$$

which we shall also denote by $\widehat{T}_{t}$.

\section{§3. Scattering theory of the Wigner-Weisskopf atom}

We will now look at the effect on the Bose field caused by interaction with the atom.

IHEOREM 3.1 For all $k \in \mathcal{K}(\mathbf{C})$ and $x \in \mathcal{K}\left(M_{2}\right)$ the following four limits exist:

$$
\begin{aligned}
\Omega_{ \pm} k: & =\lim _{t \rightarrow \pm \infty} \widehat{T}_{t} \circ \sigma_{-t}(1 \otimes k), \\
\Phi_{ \pm}(x): & =\lim _{t \rightarrow \pm \infty} \sigma_{t} \circ \widehat{T}_{-t}(x) .
\end{aligned}
$$

They define unitary maps $\Omega_{ \pm}: \mathcal{H}_{\beta} \rightarrow M_{2} \otimes \mathcal{H}_{\beta}$ and $\Phi_{ \pm}: M_{2} \otimes \mathcal{H}_{\beta} \rightarrow \mathcal{H}_{\beta}$ where $\Phi_{ \pm}=\Omega_{ \pm}^{-1}$.

Proof: Clearly, proofs for $\Omega_{+}$and $\Phi_{+}$will suffice. Write, for $k \in \mathcal{K}(\mathbf{C})$ and $x \in \mathcal{K}\left(M_{2}\right)$ :

$$
\begin{gathered}
k_{t}=\widehat{T}_{t} \circ \sigma_{-t}(1 \otimes k)=u_{t}^{*} *(1 \otimes k) * u_{t} ; \\
x_{t}=\sigma_{t} \circ \widehat{T}_{-t}(x)=u_{t} * x * u_{t}^{*} .
\end{gathered}
$$

Since $k$ is in $\mathcal{K}(\mathbf{C})$, it is of bounded support on $\nabla$; thus there exists a $T$ such that on $[T, \infty)$ the process $t \mapsto k_{t}$ is adapted. Hence $J_{+}^{\dagger} k=J_{-}^{\uparrow} k=0$ on $[T, \infty)$. Using the quantum Itô formula one calculates that for $t \geq T: J_{+}^{\downarrow} k=J_{-}^{\downarrow} k=D k=0$. Thus $t \mapsto k_{t}$ is constant for $t \geq T$ and,

$$
\Omega_{+} k=u_{T}^{*} *(1 \otimes k) * u_{T^{\prime}} .
$$

Isometry of $\Omega_{+}$follows the unitarity of $\widehat{T}_{t}$ and $\sigma_{t}$.

In order to discuss the second limit $\Phi_{+}$, let $Q$ be the orthogonal projection onto $1 \otimes \mathcal{H}_{\beta}$ in the Hilbert space $M_{2} \otimes \mathcal{H}_{\beta}$, given by

$$
Q y(\sigma)=\varphi_{\beta}(y(\sigma)) \cdot \mathbf{1} .
$$


A theorem of Kümmerer and Maassen [9] asserts that $\Phi_{+}$exists and is isometric provided that for all $x=x_{0} \otimes 1$ with $x_{0} \in M_{2}$ there is a $t>0$ such that $Q x_{t} \neq 0$. Now,

$$
\left\|Q x_{t}\right\|^{2}=\int_{\sigma \in \nabla}\left|\varphi_{\beta}\left(x_{t}(\sigma)\right)\right|^{2} \mu(d \sigma) .
$$

In view of the continuity of $(\sigma, t) \mapsto x_{t}(\sigma)$ on $\nabla_{0} \times(0, \infty)$ we need only find a single $t>0$ and $\sigma$ in some $\nabla^{i, j}$ for which $\varphi_{\beta}\left(x_{t}(\sigma)\right) \neq 0$, to obtain our result. Now, for each $x_{0} \in M_{2}$ the process $t \mapsto x_{t}$ given above is adapted. Using the quantum Itô formula we find that this process satisfies the quantum stochastic differential equations (1.4), where $L_{+}=[v, \cdot], L_{-}=-\left[v^{+}, \cdot\right]$ and $L_{0}$ is given by (2.1). Therefore, by Theorem 1.2 we find that,

$$
x_{t}(\sigma)=T_{t-s_{n}} \circ L^{\varepsilon_{n}} \circ \ldots \circ T_{s_{2}-s_{1}}^{\prime} \circ L^{\varepsilon_{1}} \circ T_{s_{1}}\left(x_{0}\right) .
$$

Take $\sigma=(\mathbf{0}, \varepsilon)$ then,

$$
\lim _{t \downarrow 0} \varphi_{\beta}\left(x_{t}(\sigma)\right)=\varphi_{\beta}\left(L^{\varepsilon_{n}} \ldots L^{\varepsilon_{1}}\left(x_{0}\right)\right) .
$$

We need now only show that for each $x_{0} \in M_{2}$ we can find a nonzero expression of this form. Let

$$
x_{0}=\left(\begin{array}{ll}
x_{11} & x_{12} \\
x_{21} & x_{22}
\end{array}\right) \text {. }
$$

If $x_{12} \neq 0$, take $\sigma=(0,+1)$, then $\varphi_{\beta}\left(x_{t}(\sigma)\right) \rightarrow x_{12} /(a+b) \neq 0,(t \downarrow 0)$;

If $x_{21} \neq 0$, take $\sigma=(0,-1)$, then $\varphi_{\beta}\left(x_{t}(\sigma)\right) \rightarrow x_{21} /(a+b) \neq 0,(t \downarrow 0)$;

If $\varphi_{\beta}(x) \neq 0$, take $\sigma=\phi$, then $\varphi_{\beta}\left(x_{t}(\sigma)\right) \rightarrow \varphi_{\beta}(x) \neq 0,(t \downarrow 0)$;

If $x_{11} \neq x_{22}$, take $\sigma=\{(0,+1),(0,-1)\}$, then $\varphi_{\beta}\left(x_{t}(\sigma)\right) \rightarrow\left(x_{11}-x_{22}\right) /(a+b) \neq 0,(t \downarrow 0)$.

Define the scattering operator $S: \mathcal{H}_{\beta} \rightarrow \mathcal{H}_{\beta}$ by

$$
S:=\Omega_{-}^{-1} \Omega_{+} \text {. }
$$

Before proceeding we will define a useful computational tool, the time reversal operator $R: \mathcal{K}\left(M_{2}\right) \rightarrow \mathcal{K}\left(M_{2}\right)$ given by,

$$
(R x)(\sigma)=x(-\sigma)
$$

LEMMA 3.2. For each $x, y \in \mathcal{K}\left(M_{2}\right)$ and $t \geq 0$,

(i) $R^{2}=i d$ and $R \circ \sigma_{t}=\sigma_{-t} \circ R$,

(ii) $R(x * y)=R x * R y$ and $R x^{*}=(R x)^{*}$,

(iii) $R\left(u_{t}\right)=\sigma_{-t}\left(u_{t}^{*}\right)$,

(iv) $R \widehat{T}_{t} R=\widehat{T}_{t}$,

(v) $\Omega_{-}=R \Omega_{+} R$.

Proof: A simple calculation; for (iii) use the explicit expression for $u_{t}$ given in Theorem 1.2. 


\section{§3.1. The fluorescent spectrum}

Let $g \in C_{0}^{\infty}(\mathbf{R}, \mathbf{C})$ and let $a_{g} \in \mathcal{H}_{\beta}$ be the annihilation kernel,

$$
a_{g}(\sigma)= \begin{cases}\overline{g(t)} & \text { if } \sigma=\left\{t^{-}\right\} \\ 0 & \text { otherwise. }\end{cases}
$$

Let $A_{g}^{\text {in }}$ and $A_{g}^{\text {out }}$ be the operators of *-multiplication by the kernels $a_{g}$ and $S a_{g}$ respectively.

We want to look at radiation detected in the field mode $g$ while our system is subjected to an intense electromagnetic field $f$. We must thus look at our observables $A_{g}^{\text {in }}$ and $A_{g}^{\text {out }}$ in the Weyl state $\chi_{f}: X \mapsto\left\langle w_{f}, X w_{f}\right\rangle$. The strength of the scattered field produced in this state is given by,

$$
\begin{aligned}
I(f, g) & =\left\|\left(A_{g}^{\text {out }}-A_{g}^{\text {in }}\right) w_{f}\right\|^{2}=\left\langle w_{f},\left(A_{g}^{\text {out }}-A_{g}^{\text {in }}\right)^{*}\left(A_{g}^{\text {out }}-A_{g}^{\text {in }}\right) w_{f}\right\rangle \\
& \left.=\left(w_{f}^{*} *\left((S-\mathbf{1}) a_{g}\right)^{*} *\left((S-1) a_{g}\right)\right) * w_{f}\right)(\phi) .
\end{aligned}
$$

The reason for considering this expression and not, for instance, the incoherent spectrum,

$$
I^{\prime}(f, g)=\left\|A_{g}^{\text {out }} w_{f}\right\|^{2}-\left\|A_{g}^{\text {in }} w_{f}\right\|^{2},
$$

is that we want to describe sideways, not forward scattering; cf. for instance [19].

We quote a useful lemma from [14].

LEMMA 3.3. For all $k \in \mathcal{K}(\mathbf{C})$,

$$
\Omega_{+} k=1 \otimes k+\int_{0}^{\infty} \widehat{T}_{t} \circ \sigma_{-t}\left(v^{+} \otimes\left(. J_{+}^{\downarrow} k\right)_{t}+v \otimes\left(J_{-}^{\downarrow} k\right)_{t}\right) d t .
$$

$\Omega_{-}$is obtained by conjugating $\Omega_{+}$with the time-reversal operator $R$.

Proof: If we put $k_{t}=\widehat{T}_{t} \circ \sigma_{-t}(k)=u_{t}^{*} *(1 \otimes k) * u_{t}$, then we find that $\left(J_{\varepsilon}^{\downarrow}-J_{\varepsilon}^{\dagger}\right)(k)=0$ for $\varepsilon= \pm 1$, using Proposition 1.1.(a); and, using Proposition 1.1.(b),

$$
(D k)_{t}=\widehat{T}_{t} \circ \sigma_{-t}\left(v^{+} \otimes\left(J_{+}^{\downarrow} k\right)_{t}+v \otimes\left(J_{-}^{\downarrow} k\right)_{t} .\right.
$$

The statement follows from the observation that

$$
\Omega_{+} k=\mathbf{1} \otimes k+\lim _{t \rightarrow \infty}\left(k_{t}-\mathbf{1} \otimes k\right)
$$

\section{§4. The two-time correlation function}

In computing the fluorescent spectrum $I(f, g)$ it turns out to be natural to study the coupled system of atom and field in the state

$$
\psi_{f}=\chi_{f} \circ \Omega_{-}^{-1} .
$$

This may be regarded as the state of the coupled system after having been acted upon by the laser field since time $-\infty$. We consider the two-time correlation function,

$$
G_{f}(s, t)=\psi_{f}\left(\widehat{T}_{s}\left(v^{+}\right) \widehat{T}_{t}(v)\right) \text {. }
$$


The following theorem connects $G_{f}$ with the fluorescent spectrum $I(f, g)$ and thus motivates the above definitions.

THFOREM 4.1. The fluorescent intensity $I(f, g)$ in the field mode $g \in C_{0}^{\infty}(\mathbf{R}, \mathbf{C})$ of $a$ two-level Wigner-Weisskopf atom, irradiated by the field $f$, is given by,

$$
I(f, g)=\int_{-\infty}^{\infty} \int_{-\infty}^{\infty} G_{f}(s, t) g(s) \overline{g(t)} d s d t
$$

Remark: If the state $\chi_{f}$ on $\mathcal{N}_{B}$ were translation invariant (which is equivalent to the state $\psi_{f}$ on $M_{2} \otimes \mathcal{N}_{\beta}$ being stationary for the flow $\left.\widehat{T}\right)$, then $G_{f}(s, t)$ would depend on $s$ and $t$ only via their difference $t-s$, and we would have,

$$
I(f, g)=\frac{1}{2 \pi} \int_{-\infty}^{\infty}|\widehat{g}(\nu)|^{2} \widehat{G}_{f}(\nu) d \nu,
$$

where $\widehat{G}_{f}$ denotes the Fourier transform of $t \mapsto G_{f}(0, t)$. Note that $\nu \rightarrow|\widehat{g}(\nu)|^{2}$ describes the spectral profile of our detector. That the fluorescent spectrum is the Fourier transform of this autocorrelation function is a well-known rule in quantum optics, and forms the starting point in the work of Mollow on the dynamical Stark effect [15].

That $G_{f}(s, t)$ depends only on $t-s$ is equivalent to $\psi_{f}$ being $\widehat{T}$-invariant on the von Neumann algebra $\mathcal{D}$ generated by $\left\{\widehat{T}_{s}\left(v^{+}\right) \widehat{T}_{t}(v) \mid s, t \in \mathbf{R}\right\}$. This again is equivalent to $\chi_{f}$ being translation invariant on $\Omega_{-}^{-1} \mathcal{D}$, an impossibility for a nonzero $f \in L^{2}(\mathbf{R})$. In a generalised sense however, the plane waves $f(t)=A \mathrm{e}^{i \omega t}$ (which are not in $L^{2}(\mathbf{R})$ ) will turn out to meet this requirement. Since a plane wave is a good description for a laser beam, shone on the atom, we are interested in giving meaning to $G_{f}$ for such $f$ 's. (cf. $\S 5)$.

Proof of theorem 4.1: The proof is a simple application of Itô's formula. First note that,

$$
(S-1) a_{g}=\Omega_{-}^{-1}\left(\Omega_{+}-\Omega_{-}\right) a_{g}=\Omega_{-}^{-1}\left(\Omega_{+}-R \Omega_{+} R\right) a_{g} .
$$

Since $a_{g} \in \mathcal{K}(\mathbf{C})$ and $\left(J_{-}^{\downarrow} a_{g}\right)_{t}=\overline{g(t)} \delta_{\phi}$ we can apply Lemma 3.3 to give,

$$
(S-1) a_{g}=\Omega^{-1}\left(\int_{-\infty}^{\infty} \widehat{T}_{t}(v) \overline{g(t)} d t\right) .
$$

As $\Omega_{-}$is a ${ }^{*}$-isomorphism, it follows that

$$
\begin{aligned}
I(f, g) & =\chi_{f}\left((S-\mathbf{1}) a_{g}^{*} *(S-\mathbf{1}) a_{g}\right) \\
& =\chi_{f} \circ \Omega_{-}^{-1}\left(\int_{-\infty}^{\infty} \widehat{T}_{s}\left(v^{+}\right) g(s) d s * \int_{-\infty}^{\infty} \widehat{T}_{t}(v) \overline{g(t)} d t\right)
\end{aligned}
$$




$$
=\int_{-\infty}^{\infty} \int_{-\infty}^{\infty} \psi_{f}\left(\widehat{T}_{s}\left(v^{+}\right) * \widehat{T}_{t}(v)\right) g(s) \overline{g(t)} d s d t
$$

Note that $G_{f}$ has the symmetries,

$$
G_{f}(s, t)=\overline{G_{f}(t, s)}(s, t \in \mathbf{R}) ; \quad G_{f}(s, t)=G_{\sigma_{-s} f}(0, t-s),(t \geq s),
$$

where $\sigma_{-s} f(r)=f(r+s)$. In view of this we need only calculate $G_{f}(0, t)$ for $t \geq 0$.

Now, for $t \geq 0$ and $f \in L^{2}(\mathbf{R})$, let us draw the diagram:

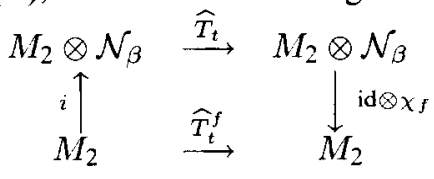

I.e. $T_{t}^{f}(x):=\left(\mathrm{id} \otimes \chi_{f}\right) \circ \widehat{T}_{t} \circ i(x)$. Note that, in terms of kernels,

$$
i(x)=x \cdot \delta_{\phi} \quad \text { and } \quad\left(\text { id } \otimes x_{f}\right)(k)=w_{f}^{*} * k * w_{f}(\phi),
$$

for each $x \in M_{2}$ and $k \in M_{2} \otimes \mathcal{N}_{\beta}$. We thus obtain a family $\left(T_{t}^{f}\right)_{t \geq 0}$ of completely positive maps $M_{2} \rightarrow M_{2}$, which can be regarded as the evolution of the Wigner-Weisskopf atom in the laser field $f^{+}=\left.f\right|_{[0, \infty)}$, provided that nothing has happened yet before time 0 . The map $T_{t}^{f}$ depends only on the behaviour of $f$ on $[0, t]$.

Remark: The projection id $\otimes \chi_{f}$ occurring in the diagram is the conditional expectation with respect to $\varphi_{\beta} \otimes \chi_{f}$. This may not seem a natural choice, since we are interested in a correlation with respect to the state $\psi_{f}$. However, unless $f=0$ no conditional expectation $E_{f}$ onto $M_{2}$ compatible with $\psi_{f}$ exists. (" $E_{f}$ compatible with $\psi_{f}$ " means that $\left.\psi_{f} \circ i \circ E_{f}=\psi_{f}\right)$. A fortiori $\left(M_{2} \otimes \mathcal{N}_{\beta}, \widehat{T}_{t}, \psi_{f} ; i\right)$ is not a Markov process.

The following lemma employs the Markov property of the unperturbed process $\left(M_{2} \otimes \mathcal{N}_{\beta}, \widehat{T}_{t}, \chi_{\beta} ; i\right)$.

LEMMA 4.2. Let $f \in C_{0}^{\infty}(\mathbf{R}, \mathbf{C})$ be decomposed as $f^{+} \oplus f^{-}$in the decomposition $L^{2}(\mathbf{R}) \oplus L^{2}\left(\mathbf{R}^{+}\right)$of $L^{2}(\mathbf{R})$. Then for all $t \geq 0$ the limil

$$
\lim _{s \rightarrow \infty} T_{s}^{\sigma_{s} f^{-}}:=\varphi_{f^{-}} \cdot \mathbf{1}
$$

exists. Moreover,

$$
G_{f}(0, t)=\varphi_{f}\left(v^{+} T_{t}^{f^{+}}(v)\right)
$$

Proof: The von Neumann algebras $\mathcal{N}_{\beta}$ and $M_{2} \otimes \mathcal{N}_{\beta}$ have respective decompositions $\mathcal{N}_{\beta}^{-} \otimes \mathcal{N}_{\beta}^{+}$and $\mathcal{N}_{\beta}^{-} \otimes M_{2} \otimes \mathcal{N}_{\beta}^{+}$, where $\mathcal{N}_{\beta}^{ \pm}$consist of (multiplication by) kernels supported on the power sets of $\mathbf{R}^{ \pm}$. The decomposition $f=f^{-} \oplus f^{+}$gives rise to a decomposition of the Weyl state $w_{f}=w_{f} \otimes w_{f}^{+}$on $\mathcal{N}_{\beta}=\mathcal{N}_{\beta}^{-} \otimes \mathcal{N}_{\beta}^{+}$. Since $\Omega_{-}^{-1}$ acts non-trivially only on the first factor in the decomposition $\left(\mathcal{H}_{\beta}^{-} \otimes M_{2}\right) \otimes \mathcal{H}_{\beta}^{+}$we must have,

$$
\psi_{f}=\psi_{f}-\otimes \chi_{f}+
$$


On the other hand, the observable $v^{+} \widehat{T}_{t}(v)$ for positive $t$ lies entirely in $\mathbf{1}_{\mathcal{N}^{-}} \otimes M_{2} \otimes \mathcal{N}_{\beta}^{+}$. If we define a state $\varphi_{f^{-}}$on $M_{2}$ by,

$$
\varphi_{f^{-}}(x)=\psi_{f^{-}}\left(\mathbf{1}_{\mathcal{N}_{\beta}^{-}} \otimes x\right),
$$

then we can write for $t \geq 0$,

$$
\begin{aligned}
G_{f}(0, t) & =\psi_{f}\left(i\left(v^{+}\right) \widehat{T}_{\imath} \circ i(v)\right)=\psi_{f^{-}} \otimes \chi_{0^{+}}\left(W\left(f^{-}\right)^{*} i\left(v^{+}\right) \widehat{T}_{t}(i(v)) W(f)\right) \\
& =\varphi_{f^{-}}\left(w_{f^{+}}^{*} * v^{+} \widehat{T}_{t}(v) * w_{f^{+}}(\phi)\right)=\varphi_{f}-\left(v^{+} T_{t}^{f}(v)\right) .
\end{aligned}
$$

Now, by Theorem 3.1 we have for all $x \in M_{2}$,

$$
\begin{aligned}
\lim _{t \rightarrow \infty} T_{t}^{\sigma_{t} f}(x) & =\lim _{t \rightarrow \infty}\left(\mathrm{id} \otimes \chi_{\sigma_{t} f}\right) \circ \widehat{T}_{t} \circ i(x)=\lim _{t \rightarrow \infty}\left(\mathrm{id} \otimes \chi_{f}\right) \circ \sigma_{-t} \circ \widehat{T}_{t} \circ i(x) \\
& =\left(\chi_{f} \circ \Omega_{-}^{-1} \circ i(x)\right) \cdot \mathbf{1}=\psi_{f}\left(x \otimes \mathbf{1}_{\mathcal{N}}\right) \cdot \mathbf{1}=\varphi_{f-}(x) \cdot \mathbf{1} .
\end{aligned}
$$

It remains to compute the action of $T_{t}^{f}, f \in C_{0}^{\infty}(\mathbf{R}, \mathbf{C})$, on elements of $M_{2}$. We tackle this problem by again making use of the non-adapted Itô calculus.

LEMMA 4.3. Let $f \in C_{0}^{\infty}(\mathbf{R}, \mathbf{C})$ and $t \geq 0$. Then, for each $x \in M_{2}$,

$$
\frac{d}{d t} T_{t}^{f}(x)=T_{i}^{f}\left(L_{0}(x)+\left[f(t) v^{+}-\overline{f(t)} v, x\right]\right) .
$$

Proof: For $t \geq 0$ define $K_{t}^{f}: M_{2} \rightarrow \mathcal{K}\left(M_{2}\right)$ by

$$
K_{t}^{f}(x)=w_{f}^{*} * u_{f}^{*} * x u_{t} * w_{f} .
$$

(The kernel $K_{t}^{f}(x)$ corresponds to the operator $W(f)^{*} \widehat{T}_{t}(x \otimes \mathbf{1}) W(f)$.) Then the process $t \mapsto K_{t}^{f}(x), t>0$, is in $\mathcal{P}^{\mathrm{l}}\left(M_{2}\right)$, and we can apply the quantum Itô formula. Noting that

$$
\left(J_{+}^{i} w_{f}\right)_{t}=f(t) w_{f} \quad \text { and } \quad\left(J_{-}^{i} w_{f}\right)_{t}=-\overline{f(t)} w_{f},
$$

( $i=\uparrow$ or $\downarrow$ ), then using (2.2) and Proposition 1.1 we have

$$
D K_{t}^{f}(x)=K_{t}^{f}\left(L_{0}(x)+\left[f(t) v^{+}-\overline{f(t)} v, x\right]\right) .
$$

The statement follows from the fact that $T_{t}^{f}(x)=K_{t}^{f}(x)(\phi)$.

\section{Input fields which are not $L^{2}$}

We are now in a position to introduce non-square-integrable input fields $f$.

Let $f$ be in $C_{b}^{\infty}(\mathbf{R}, \mathbf{C})$, the set of smooth bounded functions $\mathbf{R} \rightarrow \mathbf{C}$. Then the definition of $T_{t}^{f}$ by the diagram in $\S 4$ loses its meaning, since in general there is no Weyl kernel $w_{f}=W(f) \Omega$ in $\mathcal{H}_{\beta}$. We take our refuge in defining $T_{t}^{f}$ by the differential equation: for $t \geq 0$ let $T_{t}^{f}: M_{2} \rightarrow M_{2}$ be the unique solution of (4.8) with initial condition $T_{0}^{f}=$ id. The time-zero state $\varphi_{f}$ - and the two-time correlation function $G_{f}(s, t)$ associated 
to the input field $f$ are then defined by the limit (4.6) (if it exists), relation (4.7) and the symmetries (4.5).

These definitions are justified by the following approximation theorem.

THEOREM 5.1. Let $\left(f_{n}\right)$ be a uniformly bounded sequence of elements of $C_{0}^{\infty}(\mathbf{R}, \mathbf{C})$ tending uniformly to $f \in C_{b}^{\infty}(\mathbf{R}, \mathbf{C})$ on compact intervals. Suppose that the limit $\lim _{t \rightarrow \infty} T_{t}^{\sigma_{t} f}$ exists. Then for all $x \in M_{2}$

(a) $\lim _{t \rightarrow \infty} T_{t}^{f_{n}}(x)=T_{t}^{f}(x)$ for all $t \geq 0$;

(b) $\lim _{n \rightarrow \infty} \varphi_{f_{n}^{-}}(x)=\varphi_{f^{-}}(x)$.

As an immediate consequence (cf. (4.7) and (4.4)), this theorem gives us also

$$
\lim _{n \rightarrow \infty} G_{f^{n}}(s, t)=G_{f}(s, t) \text { and } \lim _{n \rightarrow \infty} I\left(f_{n}, g\right)=I(f, g) .
$$

For the proof we shall make use of the following orthogonal basis $B$ of $\mathrm{M}_{2}$ :

$$
B=\left\{\mathbf{1}, \gamma v, \gamma v^{+}, q\right\}, \quad \text { where } q=\left(\begin{array}{cc}
a & 0 \\
0 & -b
\end{array}\right) \quad \text { and } \quad \gamma=a+b .
$$

Note that these are all eigenvectors of $L_{0}$ (cf. (2.1)). We can write any $x \in M_{2}$ as

$$
x=\varphi_{\beta}(x) \cdot 1+x_{21} \cdot v+x_{12} \cdot v^{+}+\left(x_{11}-x_{22}\right) \cdot q .
$$

It will be useful to consider $M_{2}$ as a Banach space in the norm

$$
\|x\|_{B}=\max \left(\left|\varphi_{3}(x)\right|, \frac{1}{\gamma}\left|x_{21}\right|, \frac{1}{\gamma}\left|x_{12}\right|,\left|x_{11}-x_{22}\right|\right) .
$$

For $z \in \mathbf{C}$ let $V(z): M_{2} \rightarrow M_{2}$ denote the operator

$$
x \mapsto\left[z v^{+}-\bar{z} v, x\right]=\left[\left(\begin{array}{cc}
0 & z \\
-\bar{z} & 0
\end{array}\right), x\right] .
$$

LEMMA 5.2. Let $t \geq 0$ and $z \in \mathbf{C}$. The norm of $V(z) T_{t}$ as an operator on the Banach space $\left(M_{2},\|\cdot\|_{B}\right)$ satisfies:

$$
\left\|V(z) T_{t}\right\| \leq 4|z| \mathrm{e}^{-\frac{1}{2} \gamma t} .
$$

Proof: The operator $V(z) T_{t}$ acts on the basis vectors as follows:

$$
\begin{aligned}
1 & \mapsto 0 ; \quad q \mapsto\left(-z \gamma v^{+}-\bar{z} \gamma v\right) \mathrm{e}^{-\gamma t} ; \\
\gamma v \rightarrow(z \cdot \mathbf{1}-2 z \cdot q) \mathrm{e}^{\left(\frac{1}{2} \gamma i \omega_{0}\right) t} \gamma v ; \quad \gamma v^{+} & \mapsto(z \cdot \mathbf{1}-2 z \cdot q) \mathrm{e}^{\left(\frac{1}{2} \gamma+i \omega_{0}\right) t} \gamma v^{+} .
\end{aligned}
$$

Therefore the largest matrix element of $V(z) T_{t}$ on the basis $B$ is smaller than $2|z| \exp \left(-\frac{1}{2} \gamma t\right)$, and never more than two of them are nonzero in one row.

Proof of theorem 5.1: Let $\left(f_{n} \in C_{0}^{\infty}(\mathbf{R}, \mathbf{C})\right)$ tend to $f \in C_{b}^{\infty}(\mathbf{R}, \mathbf{C})$ uniformly on compact sets, and suppose that $\|f\|,\left\|f_{n}\right\| \leq M$. Define

$$
S_{t}^{n}:=T_{t}^{f_{n}}-T_{t}^{f} \quad \text { and } \quad U_{t}^{n}:=T_{t}^{\sigma_{t} f_{n}}-T_{t}^{\sigma_{t} f} .
$$


Then

$$
\begin{aligned}
\frac{d}{d t} S_{t}^{n} & =T_{t}^{f_{n}} \circ L_{t}^{f_{n}}-T_{t}^{f} \circ L_{t}^{f}=S_{t}^{n} \circ L_{t}^{f_{n}}+T_{t}^{f} \circ\left(L_{t}^{f_{n}}-L_{t}^{f}\right) \\
& =S_{t}^{n} \circ L_{0}+S_{t}^{n} \circ V\left(f_{n}(t)\right)+T_{t}^{f} \circ V\left(f_{n}(t)-f(t)\right) .
\end{aligned}
$$

Treating this as a perturbation of the differential equation $\frac{d}{d t} T_{t}=T_{t} \circ L_{0}$, we obtain the integral equation

$$
S_{t}^{n}=\int_{0}^{t}\left(S_{t}^{n} \circ V\left(f_{n}(s)\right)+T_{s}^{f} \circ V\left(f_{n}(s)-f(s)\right)\right) \circ T_{t-s} d s .
$$

Now, since $T_{s}^{f}$ is completely positive, it is an operator-norm contraction. On the finite dimensional space $M_{2}$ we must therefore have $\left\|T_{s}^{f}\right\| \leq c$ for some positive constant $c$. Lemma 5.2 then permits us to estimate the integrands:

$$
\begin{aligned}
\left\|S_{t}^{n}\right\| & \leq \int_{0}^{t}\left\|S_{t}^{n}\right\| \cdot\left\|V\left(f_{n}(s)\right) T_{t-s}\right\| d s+\int_{0}^{t}\left\|T_{s}^{f}\right\| \cdot\left\|V\left(f_{n}(s)-f(s)\right) T_{t-s}\right\| d s \\
& \leq 4 \int_{0}^{t}\left\|S_{t}^{n}\right\| \cdot\left|f_{n}(s)\right| \mathrm{e}^{-\frac{1}{2} \gamma(t-s)} d s+4 c \int_{0}^{t}\left|f_{n}(s)-f(s)\right| \mathrm{e}^{\frac{1}{2} \gamma(t-s)} d s .
\end{aligned}
$$

By a fundamental lemma in the theory of linear integral equations (cf. for instance [3], it follows that:

$$
\left\|S_{t}^{n}\right\| \leq 4 c \cdot \frac{2}{\gamma} \cdot \sup _{0 \leq s \leq t}\left|f_{n}(s)-f(s)\right| \cdot \exp \left(4 M \times \frac{2}{\gamma}\right) .
$$

This proves (a). To prove (b) we replace $f$ by $\sigma_{t} f$ in (5.1) and obtain the estimate

$$
\left\|U_{t}^{n}\right\| \leq 4 c \cdot I_{n} \cdot \exp \left(4 M \cdot \frac{2}{\gamma}\right),
$$

provided that $I_{n}$ is an upper bound (uniform in $t$ ) for the integral

$$
I_{n}(t)=\int_{0}^{t}\left|f_{n}(s-t)-f(s-t)\right| \mathrm{e}^{-\frac{1}{2} \gamma(t-s)} d s .
$$

We claim that:

$$
\lim _{n \rightarrow \infty} \sup _{t \geq 0}\left\|U_{t}^{n}\right\|=0
$$

Indeed, for $\varepsilon>0$ we can choose $T$ so large that for all $t \geq 0$ :

$$
\int_{0}^{(t-T) \vee 0} 2 M \mathrm{e}^{-\frac{1}{2} \gamma(t-s)} d s\left(\leq \frac{4 M}{\gamma} \mathrm{e}^{-\frac{1}{2} \gamma T}\right)<\frac{\varepsilon}{2} .
$$


Then we may choose $N$ large enough that for $n \geq N$ :

$$
\int_{(t-T)_{v} 0}^{t}\left|f_{n}(s-t)-f(s-t)\right| \mathrm{e}^{-\frac{1}{2} \gamma(t-s)} d s \leq \frac{2}{\gamma} \cdot \sup _{-t^{\prime} \leq s \leq 0}\left|f_{n}(s)-f(s)\right|<\frac{\varepsilon}{2},
$$

so that $I_{n}(t)<\varepsilon$ for all $t \geq 0$ and $n \geq N$, and our claim is proved. Finally, since $\lim _{t \rightarrow \infty} T_{t}^{f}=\varphi_{f^{-}}$exists by assumption, and $\lim _{t \rightarrow \infty} T_{t}^{f_{n}}=\varphi_{f_{n}^{-}}$by Lemma 4.2, it follows that:

$$
\lim _{n \rightarrow \infty} \lim _{t \rightarrow \infty} U_{t}^{n}=0
$$

and therefore

$$
\lim _{n \rightarrow \infty} \varphi_{f_{n}^{-}} \cdot \mathbf{1}=\lim _{n \rightarrow \infty} \lim _{t \rightarrow \infty} T_{t}^{f_{n}}=\lim _{t \rightarrow \infty} T_{t}^{f}=\varphi_{f^{-}} \cdot \mathbf{1}
$$

\section{\$6. The dynamical Stark effect}

Let us now consider a plane input wave $f$ :

$$
f(t)=e_{A, \omega}(t):=A \mathrm{e}^{-i \omega t} .
$$

In this case $T_{t}^{f}:=T_{t}^{A, \omega}$ can be explicitly calculated in terms of its Laplace transform. For $x \in M_{2}$ let

$$
\widetilde{T}_{z}^{A, \omega}(x)=\int_{0}^{\infty} T_{t}^{A, \omega}(x) \mathrm{e}^{-z t} d t, \quad(\operatorname{Re} z>0)
$$

Proposition 6.1. For $A \in \mathbf{C}, \omega \in \mathbf{R}$ and $\operatorname{Re} z>0$ we have

$$
\begin{aligned}
& \left(\begin{array}{c}
\widetilde{T}_{z}^{A, \omega}(\mathbf{1}) \\
\tilde{T}_{z-\omega}^{A, \omega}(\gamma v) \\
\widetilde{T}_{z, \omega}^{A, \omega}\left(\gamma v^{+}\right) \\
\widetilde{T}_{z}^{A, \omega}(q)
\end{array}\right) \\
& =\frac{1}{F(z)}\left(\begin{array}{cccc}
\frac{F(z)}{z} & 0 & 0 & 0 \\
-\frac{A(z+\vartheta)(z+\gamma)}{\bar{z}} & (z+\vartheta)(z+\gamma)+2|A|^{2} & -2 A^{2} & 2 A(z+\vartheta) \\
-\frac{\bar{A}(z+\bar{\vartheta})(z+\gamma)}{\left.\right|^{2} z} & -2 \bar{A}^{2} & (z+\bar{\vartheta})(z+\gamma)+2|A|^{2} & 2 \bar{A}(z+\bar{\vartheta}) \\
|A|^{\frac{2 z}{z}} & -\bar{A}(z+\vartheta) & -A(z+\bar{\vartheta}) & (z+\vartheta)(z+\bar{\vartheta})
\end{array}\right)\left(\begin{array}{c}
1 \\
\gamma v \\
\gamma v^{+} \\
q
\end{array}\right) \text {, }
\end{aligned}
$$

where $\vartheta=\frac{1}{2} \gamma+i\left(\omega-\omega_{0}\right)$, and $F(z)=F_{A, \vartheta}(z)$ is the third-order polynomial, characteristic for the Wigner-Weisskopf atom,

$$
F(z)=(z+\vartheta)(z+\bar{\vartheta})(z+\gamma)+4|A|^{2}\left(z+\frac{1}{2} \gamma\right) .
$$

Proof: The proof is a straightforward calculation. The operator $L_{t}^{f}=L_{0}+V(f(t))$ 
takes the following form on the basis $B$ of $M_{2}$ :

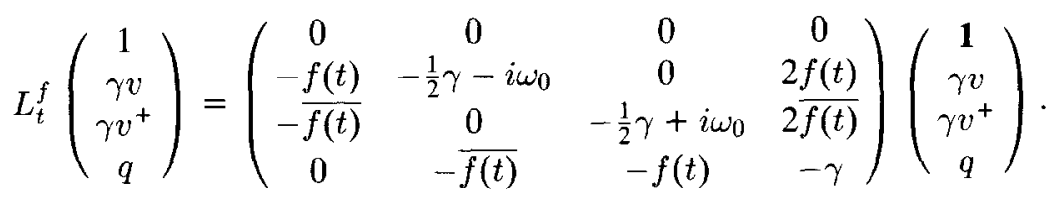

Now, taking Laplace transforms of both sides of the relation

$$
\frac{d}{d t} T_{t}^{f}(x)=T_{t}^{f} \circ L_{t}^{f}(x)
$$

and putting $T_{0}^{f}(x)=x$, we obtain for all $x \in M_{2}$ and $z \in \mathrm{C}$ with $\operatorname{Re} z>0$ :

$$
z \tilde{T}_{z}^{f}(x)-\int_{0}^{\infty} T_{t}^{f} \circ L_{t}^{f}(x) \mathrm{e}^{-z t} d t=x
$$

Then, putting $f(t)=A \mathrm{e}^{-i \omega t},(A \in \mathbf{C}, \omega \in \mathbf{R})$, and substituting the elements of $B$ for $x$, we obtain

$$
\left(\begin{array}{cccc}
z & 0 & 0 & 0 \\
A & z+\bar{\vartheta} & 0 & -2 A \\
\bar{A} & 0 & z+\vartheta & -2 \bar{A} \\
0 & \bar{A} & A & z+\gamma
\end{array}\right)\left(\begin{array}{c}
\widetilde{T}_{z}^{A, \omega}(\mathbf{1}) \\
\widetilde{T}_{z-i \omega}^{A, \omega}(\gamma v) \\
\widetilde{T}_{z, \omega}^{A+i \omega}\left(\gamma v^{+}\right) \\
\widetilde{T}_{z}^{A, \omega}(q)
\end{array}\right)=\left(\begin{array}{c}
1 \\
\gamma v \\
\gamma v^{+} \\
q
\end{array}\right)
$$

One solves this system by inverting the matrix.

Corollary 6.2. For $x \in M_{2}, A \in \mathbf{C}$ and $\omega \in \mathbf{R}$ :

$$
\lim _{t \rightarrow \infty} T_{t}^{\sigma_{t} e_{A, \omega}}(x)=\operatorname{tr}\left(\varrho_{A, \omega} x\right) \cdot \mathbf{1},
$$

where

$$
\varrho_{A, \omega}=\frac{1}{(a+b)}\left(\begin{array}{cc}
b+\frac{|A|^{2}}{2|A|^{2}+|\vartheta|^{2}} & -\frac{A \vartheta}{2|A|^{2}+|\vartheta|^{2}} \\
-\frac{A \vartheta}{2|A|^{2}+|\vartheta|^{2}} & a-\frac{|A|^{2}}{2|A|^{2}+|\vartheta|^{2}}
\end{array}\right)
$$

Remark: Note that, since $a-b=1$, we have

$$
\lim _{|A| \rightarrow \infty} \varrho_{A, \omega}=\frac{1}{2}\left(\begin{array}{ll}
1 & 0 \\
0 & 1
\end{array}\right) .
$$

This means that for very large laser field strengths upper and lower level are equally densely populated, and the polarisation (and consequently the fluorescence) disappears.

Proof: Define $n: B \rightarrow\{-1,0,1\}$ by

$$
n(\mathbf{1})=n(q)=0 ; \quad n(\gamma v)=1, \quad n\left(\gamma v^{+}\right)=-1 .
$$


Fix $\omega \in \mathbf{R}$ and call the $B \times B$-matrix occurring in Proposition 6.1: $Q(a, z)=$ $\left(Q_{x, y}(A, z)\right)_{x, y \in B}$. Then Proposition 6.1 says that for all $x \in B$,

$$
\widetilde{T}_{z-i n(x) \omega}^{A, \omega}(x)=\sum_{y \in B} Q_{x, y}(A, z) y .
$$

Now note that $\sigma_{t} e_{A, \omega}=e_{A e^{i \omega t}, \omega}$ and that

$$
Q_{x, y}\left(A \mathrm{e}^{i \omega t}, z\right)=\mathrm{e}^{i \omega(n(x)-n(y))} Q_{x, y}(A, z) .
$$

By the Fourier inversion theorem it follows that for $x \in B$ and $\lambda>0$,

$$
\begin{aligned}
T_{t}^{\sigma_{t} e_{A, \omega}}(x) & =T_{t}^{A \mathrm{e}^{i \omega t}, \omega}(x)=\int_{\lambda+i \mathbf{R}} \widetilde{T}_{z}^{A \mathrm{e}^{i \omega t}, \omega}(x) \mathrm{e}^{z t} \frac{d z}{2 \pi i} \\
& =\int_{\lambda+i \mathbf{R}} \widetilde{T}_{z-i n(x) \omega}^{A \mathrm{e}^{i \omega t}, \omega}(x) \mathrm{e}^{(z-i n(x) \omega) t} \frac{d z}{2 \pi i} \\
& =\sum_{y \in B} \int_{\lambda+i \mathbf{R}} Q_{x, y}\left(A \mathrm{e}^{i \omega t}, z\right) y \mathrm{e}^{(z-i n(x) \omega) t} \frac{d z}{2 \pi i} \\
& =\sum_{y \in B} \int_{\lambda+i \mathbf{R}} Q_{x, y}(A, z) y \mathrm{e}^{(z-i n(y) \omega) t} \frac{d z}{2 \pi i} \\
& =\sum_{y \in B} \int_{\lambda+i \mathbf{R}} Q_{x, y}(A, z+i n(y) \omega) y \mathrm{e}^{z t} \frac{d z}{2 \pi i} .
\end{aligned}
$$

Therefore,

$$
\lim _{t \rightarrow \infty} T_{t}^{\sigma_{t} e_{A, \omega}}(x)=\sum_{y \in B} \operatorname{Re} s_{-i n(y) \omega}\left(Q_{x, y}(A, \cdot)\right) y=\operatorname{Re} s_{0}\left(Q_{x, \mathbf{1}}(A, \cdot)\right) \mathbf{1},
$$

The last step can be made because $F$ has all its roots in the region $\{z \in \mathbf{C} \mid-\gamma \leq$ $\left.\leq \operatorname{Re} z \leq-\frac{1}{2} \gamma\right\}$, so that $Q(A, \cdot)$ has nonzero residues only in its first column. Thus we find $\lim _{t \rightarrow \infty} T_{t}^{\sigma_{t} e_{A, \omega}}(x)=\varphi_{A, \omega}(x) \cdot \mathbf{1}$, where

$$
\begin{gathered}
\varphi_{A, \omega}(\mathbf{1})=1 ; \quad \varphi_{A, \omega}(q)=\frac{|A|^{2}}{2|A|^{2}+|\vartheta|^{2}} ; \\
\varphi_{A, \omega}(\gamma v)=\frac{A \vartheta}{2|A|^{2}+|\vartheta|^{2}} ; \quad \varphi_{A, \omega}\left(\gamma v^{+}\right)=\frac{\overline{A \vartheta}}{2|A|^{2}+|\vartheta|^{2}} .
\end{gathered}
$$

Writing $\varphi_{A, \omega}(x)=\operatorname{tr}\left(\varrho_{A, \omega} x\right)$ we find the matrix $\varrho_{A, \omega}$ given in the statement.

Corollary 6.3. We have for all $\omega \in \mathbf{R}, A \in \mathbf{C}$ and $\operatorname{Re} z>0$,

$$
\begin{aligned}
& \widetilde{G}_{A, \omega}(z-i \omega) \\
& =\frac{1}{F(0) F(z)}\left(1+\frac{1}{z}(z+\vartheta) \bar{\vartheta}|A|^{2}+\left((z+\gamma)(z+\vartheta)+2|A|^{2}\right)\left(\gamma|A|^{2}+(\gamma-1)|\vartheta|^{2}\right)\right) .
\end{aligned}
$$


Proof: Note that

$$
\widetilde{G}_{A, \omega}(z-i \omega)=\varphi_{A, \omega}\left(v^{+} \widetilde{T}_{z-i \omega}^{A, \omega}(v)\right) .
$$

Using Proposition 6.1 and the expressions (6.1) and (6.2) we calculate the right-hand side.

With the benefit of hindsight we can now see that $G_{A, \omega}(s, t)$ depends on $s$ and $t$ only via $t-s$. Indeed, since $G_{A, \omega}$ does not depend on the phase of $A$, we have for $t \geq s$ by (4.5)

$$
G_{A, \omega}(s, t)=G_{A \mathrm{e}^{i \omega t}, \omega}(0, t-s)=G_{A, \omega}(0, t-s) .
$$

Since $G_{A, \omega}(s, t)=\overline{G_{A, \omega}(t, s)}$, the statement goes through for $t<s$ as well. We shall henceforth consider $G_{A, \omega}$ as a function of one variable.

We will now examine (6.3) more closely. Since the roots of the polynomial $F$ have their real parts lying between $-\gamma$ and $-\frac{1}{2} \gamma$, the only contribution to the asymptotic form of $G_{A, \omega}$ comes from the pole at $z=0$. The residue of this pole is

$$
\frac{|A \vartheta|^{2}}{F(0)^{2}}=\frac{|A \vartheta|^{2}}{2|A|^{2}+|\vartheta|^{2}}=\left|\varphi_{A, \omega}(v)\right|^{2} .
$$

This contribution to $G_{A, \omega}$ is the coherent plane wave

$$
G_{A, \omega}^{\mathrm{coh}}(t)=\left|\varphi_{A, \omega}(v)\right|^{2} \mathrm{e}^{-i \omega t} .
$$

Write $G_{A, \omega}^{\mathrm{inc}}=G_{A, \omega}-G_{A, \omega}^{\mathrm{coh}}$, the remaining incoherent component of the correlation function.

We now collect our results.

Proposition 6.4. For all $g \in C_{0}^{\infty}(\mathbf{R}, \mathbf{C})$,

$$
I\left(e_{A, \omega}, g\right)=\frac{1}{2 \pi} \int_{-\infty}^{\infty}|\widehat{g}(\nu)|^{2} I_{A, \omega}(d \nu),
$$

where $I_{A, \omega}$ is the measure on $\mathbf{R}$ given by

$$
\begin{gathered}
I_{A, \omega}(d \nu)=\left|\varphi_{A, \omega}(v)\right|^{2} \delta_{\omega}(d \nu)+I_{A, \omega}^{\mathrm{inc}}(\nu) d \nu ; \\
I_{A, \omega}^{\mathrm{inc}}(\nu)=2 \operatorname{Re} \widetilde{G}_{A, \omega}^{\mathrm{inc}}(i \nu) .
\end{gathered}
$$

We shall only evaluate the spectral density $I_{A, \omega}$ in the resonant case, i.e., for $\omega=\omega_{0}$. We will also assume that the field is sufficiently strong for $\Omega^{2}:=4|A|^{2}-\frac{1}{16} \gamma^{2}$ to be positive. (A discussion of the general case can be found in [15].)

At resonance $F$ has the simple factorisation

$$
F(z)=\left(z+{ }_{2}^{1} \gamma\right)\left(z+i \Omega+{ }_{4}^{3}\right)\left(z-i \Omega+\frac{3}{4}\right) .
$$

Substituting into (6.3) we find after a long reduction,

$$
I_{A, \omega}^{\mathrm{inc}}(\nu)=\frac{\gamma\left(F(0)-\frac{1}{4} \gamma^{2}\right)}{4 F(0)\left((\omega-\nu)^{2}+\frac{1}{4} \gamma^{2}\right)}+\frac{-C(\omega-\nu-\Omega)+\frac{3}{4} \gamma D}{(\omega-\nu-\Omega)^{2}+\left(\frac{3}{4} \gamma\right)^{2}}+\frac{C(\omega-\nu+\Omega)+\frac{3}{4} \gamma D}{(\omega-\nu+\Omega)^{2}+\left(\frac{3}{4} \gamma\right)^{2}},
$$


where

$$
\begin{gathered}
C=\frac{\gamma\left(\frac{1}{2} \gamma\right)^{2}(\gamma-1)}{16 \Omega F(0)}+\frac{\gamma|A|^{2}}{8 \Omega F(0)}\left(\frac{(\gamma+4) F(0)-\frac{3}{2} \gamma^{2}}{F(0)}\right), \\
D=\frac{\frac{1}{4} \gamma^{2}(\gamma-1)}{4 F(0)}+\frac{\gamma|A|^{2}}{2 F(0)}\left(\frac{F(0)-\frac{1}{4} \gamma^{2}}{F(0)}\right)
\end{gathered}
$$

Equation (6.6) represents a three peaked spectrum centred at $\omega$ with side bands displaced at $\pm \Omega$; the spectrum is symmetric about the central peak and the central peak has width two-thirds that of the side peaks. For $|A| \gg \gamma$, i.e., in the limit of very strong fields,

$$
I_{A, \omega}^{\mathrm{inc}}(\nu)=\frac{\frac{1}{4} \gamma}{(\omega-\nu)^{2}+\frac{1}{4} \gamma^{2}}+\frac{\frac{3}{16} \gamma}{(\omega-\nu-2|A|)^{2}+\left(\frac{3}{4} \gamma\right)^{2}}+\frac{\frac{3}{16} \gamma}{(\omega-\nu+2|A|)^{2}+\left(\frac{3}{4} \gamma\right)^{2}}
$$

As mentioned in the introduction, this spectrum was first computed by Mollow [15] in the case of a zero-temperature Bose field. This case is recovered by putting $\gamma=1$ above. The finite-temperature dynamical Stark effect is very similar to the one at zero temperature; note however the temperature-dependent spectral line frequencies in (6.6) and the temperature-broadening, and temperature-dependent peak heights, of the three Lorentz lines in (6.7).

A further difference with the dynamical Stark effect at temperature zero is the occurrence of fluorescence already in the absence of an input field, due to the thermal background only. It has the following spectral shape:

$$
I_{0, \omega}^{\text {inc }}(\nu)=\frac{\gamma-1}{\left(\nu+\omega_{0}\right)^{2}+\frac{1}{4} \gamma^{2}}
$$

\section{REFERENCES}

[1] A. Barchielli: $J$. Phys. A20 (1987), No. 18.

[2] A. Barchielli: Lecture Notes in Mathematics 1303, Springer (1988).

[3] R. Bellman: Stability Theory of Differential Equations, Mc Graw-Hill Book Company, Inc, New York, Toronto and London (1953).

[4] H. J. Carmichael and D. F. Walls: J. Phys. B9 (1976), 1199-1220.

[5] C. S. Chang and P. Stehle: Phys. Rev. A4 (1971), 641-661.

[6] S. Ezekiel and F. Y. Wu: "Two-level atoms in an intense monochromatic field: A review of recent experimental investigations", Multiphoton Processes, ed. J.H. Eberly and P. Lambropoulos, John Wiley \& Sons, New York (1977).

[7] R. L. Hudson and J. M. Lindsay: Lecture Notes in Mathematics 1136, Springer (1985).

[8] B. Kümmerer: J. Funct. Anal. 63 (1985).

[9] B. Kümmerer and H. Maassen: preprint, Tübingen (1989).

[10] J. M. Lindsay: "Quantum Stochastic Calculus with Integral Kernels I", Proc. First World Congress of the Bernoulli Society, eds. Y. Prohorov and V. Y. Sazonov (1987).

[11] J. M. Lindsay: $A$ Quantum Stochastic Calculus, thesis, Nottingham (1985).

[12] J. M. Lindsay and H. Maassen: The Stochastic Calculus of Bose Noise, preprint, Nijmegen (1988).

[13] H. Maassen: Lecture Notes in Mathematics 1136, Springer (1985).

[14] H. Maassen: "Quantum Stochastic Calculus with Integral Kernels II: the Wigner-Weisskopf Atom”, Proc. First World Congress of the Bernoulli Society, eds. Y. Prohorov and V. Y. Sazonov (1987). 
[15] B. R. Mollow: Phys. Rev. 188 (1969), 1969-1975.

[16] B. R. Mollow: Phys. Rev. A12 (1975), 1919-1945.

[17] F. Schuda, C. R. Stroud Jr., and M. Hercher: J. Phys. B7 (1974), L198.

[18] M. E. Smithers and H. S. Freedhoff: J. Phys. B7 (1974), 2432-2435.

[19] J. R. Taylor: Scattering Theory, John Wiley \& Sons, Inc., New York (1972).

[20] V. Weisskopf and E. Wigner: Zeitschrift für Physik 63 (1930), 54. 